\title{
Expression of secreted phospholipase A2-Group IIA correlates with prognosis of gastric adenocarcinoma
}

\author{
CHENGWEI ZHANG ${ }^{1 *}$, HAIPENG YU ${ }^{2 *}$, HAIYAN XU ${ }^{3}$ and LANLAN YANG ${ }^{4}$ \\ ${ }^{1}$ Department of Anesthesiology, The Second Hospital of Jilin University, Changchun, Jilin 130041; \\ ${ }^{2}$ Department of Interventional Therapy, Tianjin Medical University Cancer Institute and Hospital, \\ National Clinical Research Center of Cancer, Key Laboratory of Cancer Prevention and Therapy, Tianjin 300060; \\ ${ }^{3}$ Department of Physiology, College of Basic Medical Sciences, Jilin University, Changchun, Jilin 130021; \\ ${ }^{4}$ Department of Biliary and Pancreatic Internal Medicine, The Second Hospital of Jilin University, \\ Changchun, Jilin 130041, P.R. China
}

Received October 26, 2014; Accepted August 17, 2015

DOI: $10.3892 / \mathrm{ol} .2015 .3736$

\begin{abstract}
The present study investigated the expression of secretory phospholipase A2-Group IIA (sPLA2-II) in gastric adenocarcinoma, in order to evaluate the correlation between sPLA2-II expression, and the clinicopathological features and prognosis of patients with gastric adenocarcinoma. Between January 2007 and April 2010, data were collected from 65 patients (44 males, 21 females; age range, 30-79 years; mean $66.7 \pm 10.7$ years). All patients exhibited a pathologically confirmed diagnosis of gastric adenocarcinoma. Endoscopic biopsy specimens of normal gastric mucosa from 11 of these patients were used as controls. Patients were subsequently followed-up at 3-month intervals, and survival data were recorded until April 2010. Expression of sPLA2-II in 65 gastric adenocarcinoma and 11 normal gastric mucosa specimens was evaluated via immunohistochemistry. A semi-quantitative method, consisting of evaluation of staining percentage and intensity, was utilized for immunohistochemical scoring, and the receiver operating characteristic curve method was applied to select a cut-off score for high and low sPLA2-II expression. The value of 8 was selected as the cut-off score, with maximum sensitivity and specificity. High sPLA2-II expression was observed in stage III/IV cases $(83.3 \% ; 40 / 48)$ and poorly differentiated cells $(94.1 \%$; 32/34), while sPLA2-II expression levels were observed to be significantly lower in stage I/II cases $(52.9 \%$; 9/17) and well and moderately differentiated cells (54.8\%; 17/31; $\mathrm{P}=0.021$ and $\mathrm{P}<0.001$, respectively). There
\end{abstract}

Correspondence to: Dr Lanlan Yang, Department of Biliary and Pancreatic Internal Medicine, The Second Hospital of Jilin University, 218 Ziqiang Street, Changchun, Jilin 130041, P.R. China E-mail:wsyhy@163.com

*Contributed equally

Key words: secretory phospholipase A2-Group IIA, gastric adenocarcinoma, immunohistochemistry, receiver operating characteristic were no significant correlations observed between sPLA2-II expression and any other clinicopathological parameters, including gender, age, tumor diameter and Helicobacter pylori infection. Patients exhibiting low sPLA2-II expression experienced significantly improved overall survival (OS) and disease-free survival (DFS), compared with those exhibiting high sPLA2-II expression ( $\mathrm{P}=0.043$ and $\mathrm{P}=0.035$, respectively). Multivariate analysis confirmed that high sPLA2-II expression may be an independent prognostic factor for OS [relative risk, 2.849; 95\% confidence interval (CI), 1.088-7.459; $\mathrm{P}=0.033$ ] and DFS (relative risk, 2.735; 95\% CI, 1.104-6.776; $\mathrm{P}=0.030$ ) in gastric adenocarcinoma. Therefore, sPLA2-II may be correlated with the histogenesis of gastric adenocarcinoma, and increased sPLA2-II expression may be an indicator of poor prognosis.

\section{Introduction}

It is well understood that tumor development is a multi-factorial, multi-stage process, during which the activation of oncogenes and inactivation of tumor-suppressor genes are significant events $(1,2)$. The progression of gastric carcinoma has been suggested to be associated with gastritis, intestinal metaplasia and dysplasia to adenocarcinoma (3). Determining the molecular markers associated with the progression and prognosis of gastric carcinoma remains a significant challenge. Phospholipase (PLA), an enzyme that hydrolyzes phospholipids into phosphatidic acid and amino alcohols, is observed mainly in murine or human cells, and mitochondrial cell membranes (4). In vivo, phospholipids frequently contain polyunsaturated fatty acids and, when released, these may be metabolized to form certain eicosanoids and associated bioactive lipid mediators (5). Following metabolism, the remaining lysophospholipids may additionally possess significant roles in various biological processes (6). Furthermore, PLA is involved in phospholipid reconstruction, pulmonary surfactant metabolism, cell signaling, host responses and the promotion of blood clotting $(7,8)$. Previous studies have indicated that the expression of secretory PLA A2-Group IIA (sPLA2-II) is associated with tumor malignancy (6-9). Thus, sPLA2-II has become the 
focus of significant attention in the field of cancer research. However, to the best of our knowledge, few studies have investigated the significance of sPLA2-II in gastric adenocarcinoma or its association with prognosis. In the present study, the clinicopathological significance of sPLA2-II in gastric adenocarcinoma was evaluated using immunohistochemistry, and the expression of sPLA2-II in gastric adenocarcinoma and normal gastric tissues was observed, in order to examine the association between SPLA2-II expression and the degree of tumor differentiation and tumor stage, and elucidate the potential value of sPLA2-II in clinical prognosis.

\section{Materials and methods}

Clinical data. Between January 2007 and April 2010, data were collected from 65 patients at the Second Hospital of Jilin University (Changchun, China) and Tianjin Medical University Cancer Hospital (Tianjin, China). The present study was approved by the Research Ethics Committee of the Second Hospital of Jilin University (Changchun, China) and written informed consent was obtained from all patients, or their family. All 65 patients (44 males, 21 females; age range, 30-79 years, mean age, $66.7 \pm 10.7$ years) possessed a pathologically confirmed diagnosis of gastric adenocarcinoma. Surgical specimens were obtained from 51 total gastrectomies and 14 partial resections of the stomach. Differentiation statuses of the gastric adenocarcinoma specimens were as follows: Well-differentiated, 14 patients; moderately differentiated, 17 patients; poorly differentiated, 34 patients. Fifty patients exhibited lymph node metastases. Endoscopic biopsy specimens of normal gastric mucosa from 11 of the patients served as controls.

Patients were followed-up at 3-month intervals, and survival data were recorded until April 2010. Patients were enrolled in the present study based on the following eligibility criteria: i) Histopathologically proven gastric adenocarcinoma; ii) disease stage diagnosed using the seventh edition of the International Union Against Cancer staging system (10) for gastric cancer; iii) at least 18 years of age; and iv) no history of previously treated cancer. Following surgery, patients received chemotherapy and/or radiotherapy according to their individual condition. Histological classifications were assigned according to the World Health Organization International Histological Classification of Tumors (current at the time of diagnosis) (11). Histological grade was evaluated and categorized as follows: Well-differentiated (Grade 1), moderately differentiated (Grade 2) and poorly differentiated (Grade 3). Helicobacter pylori infection was identified using the rapid test strips method (12).

Instruments and equipment. Instruments and equipment were provided by the Department of Pathology of Tianjin Medical University Cancer Hospital (Tianjin, China) and comprised: A microtome (HM325; Carl Zeiss AG, Oberkochen, Germany), a tissue array analyzer (MTA-1; Beecher Instruments, Sun Prairie, WI, USA), a flattening bath for paraffin sections (Leica HI1210; Leica Microsystems, Glattbrugg, Switzerland), a flattening table (Leica HI1220; Leica Microsystems), a research microscope (Olympus BX40; Olympus Corp., Tokyo, Japan) and an automatic micrograph with DP camera software (Olympus Corp.).
Reagents. Streptavidin-peroxidase kits were obtained from KPL, Inc. (Gaithersburg, MD, USA). Mouse anti-human monoclonal sPLA2-II primary antibody was obtained from Cayman Chemical Co. (cat no. 160500; Ann Arbor, MI, USA). H. pylori rapid test strips were obtained from Guangzhou Bei Siqi Diagnostic Reagents Ltd. (Tianjin, China; batch number 20070101).

Immunohistochemistry. Immunoperoxidase staining for sPLA2-II was performed manually by a board-certified lab technician, according to the manufacturer's instructions for technical and interpretative problems. Tissue was sectioned $(4 \mu \mathrm{m})$ and mounted onto positively charged glass slides (X-tra ${ }^{\circledR}$ slides; Leica Biosystems Nussloch GmbH, Nussloch, Germany). Slides were dried at $60^{\circ} \mathrm{C}$ overnight, deparaffinized and subsequently rehydrated [two washes with xylene (10 min each); two washes with $100 \%$ ethanol (3 min each); two washes with $95 \%$ ethanol (3 min each); and two washes with distilled water (2 min each)]. Endogenous peroxidase activity was blocked by incubation with $0.3 \%$ hydrogen peroxide (Wuhan Boster Biological Engineering Co., Ltd., Wuhan, China) for $15 \mathrm{~min}$. In order to retrieve antigens, tissue slides were boiled in $10 \mathrm{mM}$ citrate buffer ( $\mathrm{pH}$ 6.0; Wuhan Boster Biological Engineering Co., Ltd.) and microwave (M1-L213B; Guangdong Midea Microwave and Electrical Appliances Manufacturing Co., Ltd., Foshan, China)-treated for $10 \mathrm{~min}$. Non-specific binding was blocked by incubation with $10 \%$ normal rabbit serum (Wuhan Boster Biological Engineering Co., Ltd.) for $20 \mathrm{~min}$. Subsequently, tissue slides were incubated with monoclonal antibody against sPLA2-II (1:100 dilution; Cayman Chemical Co) overnight at $4^{\circ} \mathrm{C}$. Following five rinses with $0.01 \mathrm{~mol} / \mathrm{l}$ phosphate-buffered saline (PBS; pH 7.4; Wuhan Boster Biological Engineering Co., Ltd.) for $10 \mathrm{~min}$, primary antibody was detected using an Envision ${ }^{\mathrm{TM}}$ Detection System, according to the manufacturer's instructions (cat no. K5007; Dako, Glostrup, Denmark) for $1 \mathrm{~h}$ at room temperature. Cells were subsequently stained with 30,30-diaminobenzidine (Seebio Biotech, Inc., Shanghai, China) following another wash with PBS. Finally, sections were counterstained using Mayer's hematoxylin (Wuhan Boster Biological Engineering Co., Ltd.), dehydrated and mounted. In order to ensure accurate and reproducible staining, sections of gastric adenocarcinoma were used as a positive control, while PBS was used as a negative control.

Immunohistochemical scoring. Immunohistochemical scoring of sPLA2-II staining was performed on one batch of slides of high quality by a semi-quantitative method (13). The mean percentage of positive tumor cells was determined in $\geq 5$ areas (magnification, $\mathrm{x} 400$ ), and assigned one of the following six categories: $<10 \%$ tumor cells positively stained, 0 points; $10-40 \%, 1$ point; 40-70\%, 2 points; $\geq 70 \%, 3$ points. Staining intensity was scored as follows: No coloring, 0 points; yellow, 1 point; brown, 2 points; dark brown, 3 points. As tumors may demonstrate heterogeneous staining, the dominant staining pattern was selected when scoring the samples. The percentage of positive cells and the staining intensity were added to produce a weighted score for each sample, as follows: 0-2 points, low expression; 3-6 points, high expression (14). In the present study, sPLA2-II expression was assessed by two independent, board-certified pathologists, who were blinded to clinical follow-up data. Their conclusions were in complete agreement 
Table I. Clinicopathological features of patients and tumors $(\mathrm{n}=65)$.

\begin{tabular}{lc}
\hline Clinicopathological feature & $\begin{array}{c}\text { Patients with gastric } \\
\text { adenocarcinoma, } \mathrm{n}\end{array}$ \\
\hline Gender & 44 \\
Male & 21 \\
Female & \\
Age, years & 67 \\
$\quad$ Median & $30-79$ \\
Range & \\
Helicobacter pylori infection & 29 \\
Negative & 36 \\
Positive & \\
Tumor size, cm & 23 \\
$\leq 3$ & 42 \\
$>3$ & \\
Histological grade & \\
1 & 14 \\
2 & 17 \\
3 & 34 \\
Tumor stage & \\
I & 17 \\
II & 48 \\
\hline
\end{tabular}

${ }^{\mathrm{a}}$, well differentiated; 2 , moderately differentiated; 3 , poorly differentiated.

(percentage staining and staining intensity scores) in 58 of the cases, which suggested that the scoring method was highly reproducible. In 7 cases when the two scoring results differed, a third blinded pathologist intervened and evaluated these cases. If the third pathologist agreed with one of the previous scores, that score was used for analysis. For the single case in which six distinct scores were obtained, the three pathologists collaborated to reach an agreement on the final score.

Statistical analysis. The selection of clinically significant cut-off scores for the expression of sPLA2-II was based on receiver operating characteristic (ROC) curve analysis $(13,15)$. For each percentage score, the sensitivity and specificity for each outcome under evaluation was plotted, thus generating a ROC curve. The score that was the closest to the point with maximum sensitivity and specificity, i.e. the point $(0.0,1.0)$ on the curve, was selected as the cut-off score, resulting in the greatest number of tumors correctly classified as having a diagnosis of gastric adenocarcinoma or not. The area under the ROC curve (AUC) was calculated in order to estimate the discriminatory power of sPLA2-II over the entire range of scores for overall survival (OS) and disease-free survival (DFS). All data were assessed using SPSS 16.0 (SPSS Inc., Chicago, IL, USA). Associations between the expression of sPLA2-II and clinicopathological variables were investigated using the $\chi^{2}$ test. Survival curves were plotted using the Kaplan-Meier method, and were compared using the log-rank test. Generation and analysis of the cut-off points were
A

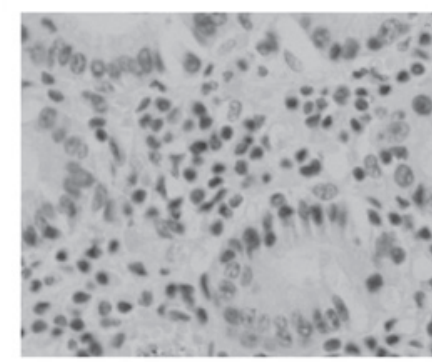

B

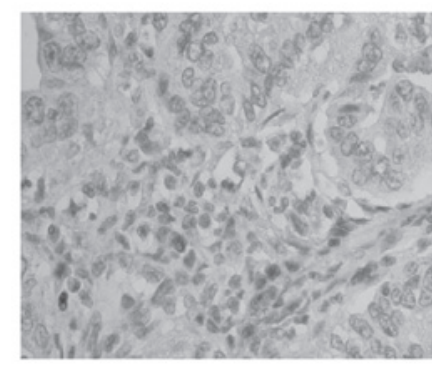

C

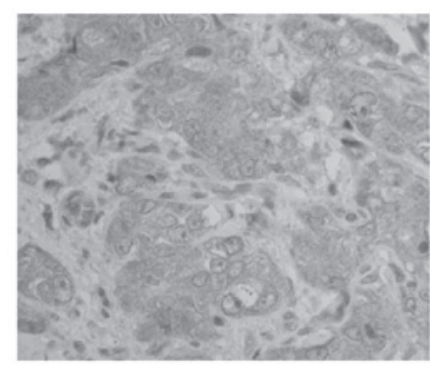

Figure 1. Representative IHC photomicrographs of cytoplasmic sPLA2-II expression in gastric adenocarcinoma tissue sections. (A) Negative sPLA2-II expression (magnification, $\mathrm{x} 400$; IHC score, 0). (B) Low sPLA2-II expression (magnification, x400; IHC score, 6). (C) High sPLA2-II expression (magnification, x400; IHC score, 12). IHC, immunohistochemistry; sPLA2-II, secretory phospholipase A-Group IIA.

performed by ROC curve. Cox proportional hazards regression was performed for multivariate analysis of prognostic factors. $\mathrm{P}<0.01$ and $\mathrm{P}<0.05$ were considered to indicate a statistically significant difference.

\section{Results}

SPLA2-II expression is associated with certain clinicopathological characteristics. The clinicopathological characteristics of the 65 cases of gastric adenocarcinoma are listed in Table I. sPLA2-II was expressed in the cytoplasm of gastric carcinoma and normal cells (Fig. 1). According to the results of the ROC curve analysis for OS and DFS, a cut-off score, with maximum sensitivity and specificity, of 8 was selected (Fig. 2). The AUC of the two ROC curves were 0.761 and 0.818 , respectively $(\mathrm{P}=0.002$ and $\mathrm{P}<0.001)$. All 65 patients were categorized into a high (49 cases, $75.4 \%$ ) or low expression group (16 cases, $24.6 \%$ ). Expression of sPLA2-II in normal gastric mucosa and gastric cancer cases are listed in Table II.

SPLA2-II expression is correlated with certain clinicopathological characteristics. Correlations between sPLA2-II expression and clinicopathological characteristics are listed in Table III. High sPLA2-II expression was observed in stage I $(83.3 \%$; $40 / 48)$ and poorly differentiated cells $(94.1 \%$; 
Table II. sPLA2-II expression in normal gastric mucosa and gastric cancer cases.

\begin{tabular}{lccrr}
\hline Tissue type & $\mathrm{n}$ & High sPLA2-II expression & Low sPLA2-II expression & P-value \\
\hline Normal gastric mucosa & 11 & 4 & 7 & 0.015 \\
Gastric adenocarcinoma & 65 & 49 & 16 & \\
\hline
\end{tabular}

sPLA2-II, secretory phospholipase A-Group IIA.

Table III. Association between the expression of sPLA2-II and clinicopathological variables.

\begin{tabular}{|c|c|c|c|c|}
\hline \multirow[b]{2}{*}{ Clinicopathological feature } & \multirow[b]{2}{*}{$\mathrm{n}$} & \multicolumn{2}{|c|}{ sPLA2-II expression } & \multirow[b]{2}{*}{ P-value } \\
\hline & & Low, n (\%) & High, n (\%) & \\
\hline Gender & & & & 0.229 \\
\hline Male & 44 & $13(29.5)$ & $31(70.5)$ & \\
\hline Female & 21 & $3(14.3)$ & $18(85.7)$ & \\
\hline Age, years & & & & 0.076 \\
\hline$\leq 65$ & 41 & $13(31.7)$ & $26(63.4)$ & \\
\hline$>65$ & 24 & $3(12.5)$ & $23(87.5)$ & \\
\hline Tumor diameter, cm & & & & 0.243 \\
\hline$\leq 3$ & 27 & $9(33.3)$ & $18(66.7)$ & \\
\hline$>3$ & 41 & $7(18.4)$ & $9(81.6)$ & \\
\hline Helicobacter pylori infection & & & & 0.332 \\
\hline Negative & 25 & $20(80.0)$ & $5(20.0)$ & \\
\hline Positive & 40 & $30(75.0)$ & $10(25.0)$ & \\
\hline Tumor stage & & & & 0.021 \\
\hline I & 48 & $8(16.7)$ & $40(83.3)$ & \\
\hline II & 17 & $8(47.1)$ & $9(52.9)$ & \\
\hline Histological grade ${ }^{a}$ & & & & $<0.001$ \\
\hline 3 & 34 & $2(5.9)$ & $32(94.1)$ & \\
\hline 1 and 2 & 31 & $14(45.2)$ & $17(54.8)$ & \\
\hline
\end{tabular}

sPLA2-II, secretory phospholipase A-Group IIA. ${ }^{\mathrm{a}} 1$, well differentiated; 2 , moderately differentiated; 3 , poorly differentiated.

32/34), while sPLA2-II expression levels were observed to be significantly lower in stage II $(52.9 ; 9 / 17)$ and well/moderately differentiated cells $(54.8 \%, 17 / 31 ; \mathrm{P}=0.021$ and $\mathrm{P}<0.001$, respectively). There were no significant correlations observed between sPLA2-II expression and any other clinicopathological parameters evaluated, including gender, age, tumor diameter and $H$. pylori infection.

Low SPLA2-II expression is associated with improved OS and DFS. At the time of the present analysis (April 2010), with a median follow-up of 509 days (range, 201-1208 days), 15 patients $(23.1 \%)$ were alive and $13(20 \%)$ also remained free of disease. Kaplan-Meier survival curves (Fig. 3) indicated that patients exhibiting low sPLA2-II expression experienced significantly improved OS and DFS compared with patients exhibiting high sPLA2-II expression $(\mathrm{P}=0.043$ and $\mathrm{P}=0.035$, respectively).

Analysis was performed using the Cox proportional hazards model in order to identify factors involved in OS
(Table IV) and DFS (Table V). Multivariate analysis confirmed that high SPLA2-II expression was an independent prognostic factor for OS [relative risk, 2.849; 95\% confidence interval (CI), 1.088-7.459, $\mathrm{P}=0.033$ ] and DFS (relative risk, 2.735; 95\% CI, 1.104-6.776, $\mathrm{P}=0.030$ ).

\section{Discussion}

In the present study, increased sPLA2-II expression was observed in gastric adenocarcinoma tissues compared with that of normal gastric tissues (Table III; Fig. 1). High sPLA2-II expression was also observed in poorly differentiated cells, and this level of expression was significantly higher than that observed in well and moderately differentiated cells. The results of the present study revealed overexpression of sPLA2-II protein in gastric adenocarcinoma tissues, which suggested an association between sPLA2-II gene activation and the occurrence of gastric adenocarcinoma. In addition, sPLA2-II expression was associated with the histopathological 
Table IV. Univariate and multivariate analyses of overall survival analysis using Cox proportional hazards regression.

A, Univariate analysis

\begin{tabular}{|c|c|c|c|c|}
\hline Parameter & Variable & Hazard ratio & $95 \%$ confidence interval & P-value \\
\hline Age, years & $\leq 65$ vs. $>65$ & 0.840 & $0.480-1.470$ & 0.542 \\
\hline Gender & Male vs. female & 0.937 & $0.524-1.637$ & 0.825 \\
\hline Tumor diameter, $\mathrm{cm}$ & $\leq 3$ vs. $>3$ & 1.329 & $0.754-2.341$ & 0.326 \\
\hline Helicobacter pylori infection & Negative vs. positive & 0.663 & $0.311-1.414$ & 0.287 \\
\hline Histological grade ${ }^{a}$ & 3 vs. 1 and 2 & 2.090 & $1.193-3.662$ & 0.010 \\
\hline Tumor stage & I vs. II & 1.774 & $0.947-3.325$ & 0.073 \\
\hline sPLA2-II expression & Low vs. high & 2.126 & $0.996-4.540$ & 0.051 \\
\hline
\end{tabular}

B, Multivariate analysis

\begin{tabular}{|c|c|c|c|c|}
\hline Parameter & Variable & Hazard ratio & $95 \%$ confidence interval & P-value \\
\hline Age, years & $\leq 65$ vs. $>65$ & 0.602 & $0.204-1.777$ & 0.358 \\
\hline Gender & Male vs. female & 1.088 & $0.390-3.036$ & 0.872 \\
\hline Tumor diameter, $\mathrm{cm}$ & $\leq 3$ vs. $>3$ & 1.343 & $0.648-2.788$ & 0.428 \\
\hline Helicobacter pylori infection & Negative vs. positive & 0.858 & $0.351-2.094$ & 0.736 \\
\hline Histological grade & 3 vs. 1 and 2 & 1.491 & $0.790-2.813$ & 0.218 \\
\hline Tumor stage & I vs. II & 3.330 & $1.432-7.744$ & 0.005 \\
\hline sPLA2-II expression & Low vs. high & 2.849 & $1.088-7.459$ & 0.033 \\
\hline
\end{tabular}

sPLA2-II, secretory phospholipase A-Group IIA. ${ }^{\mathrm{a}}$, well differentiated; 2 , moderately differentiated; 3 , poorly differentiated.

A

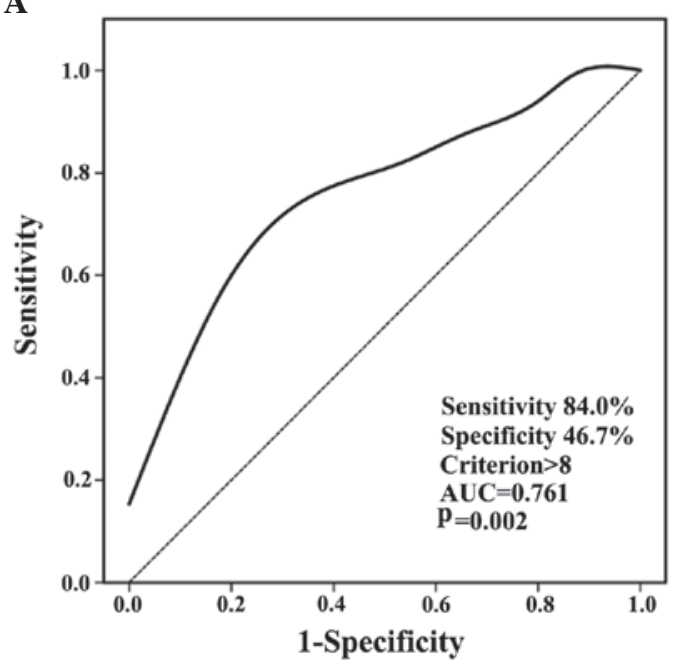

B

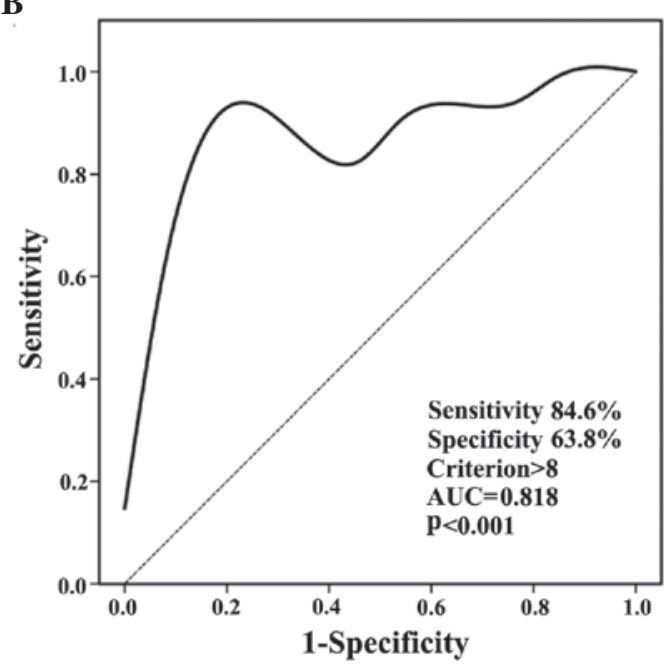

Figure 2. ROC curves for OS and DFS. (A) ROC curve analysis for OS. (B) ROC curve analysis for DFS. ROC, receiver operating characteristic; AUC, area under curve; OS, overall survival; DFS, disease-free survival.

differentiation of gastric adenocarcinoma, suggesting that SPLA2-II may be a marker for gastric adenocarcinoma. This suggested the existence of a link between sPLA2-II expression and gastric cancer progression and tumorigenesis. Furthermore, these results suggested that sPLA2-II expression may be used as a marker for prognosis and for predicting the biological behavior of gastric cancer. A previous study utilizing reverse transcription-polymerase chain reaction analysis to evaluate paired samples from 23 cases of gastric cancer, demonstrated that sPLA2-II expression in gastric cancer tissues and adjacent normal tissues differed, suggesting that sPLA2-II may possess a role in the development of gastric cancer (16).

The present study additionally identified that sPLA2-II expression in gastric adenocarcinoma was associated with H. pylori infection. The results of the present study are supported by those of previous studies (17-19). These previous 
Table V. Univariate and multivariate analyses of disease-free survival analysis using Cox proportional hazards regression.

A, Univariate analysis

\begin{tabular}{|c|c|c|c|c|}
\hline Parameter & Variable & Hazard ratio & $95 \%$ confidence interval & P-value \\
\hline Age, years & $\leq 65$ vs. $>65$ & 0.908 & $0.521-1.581$ & 0.732 \\
\hline Gender & Male vs. female & 1.012 & $0.568-1.804$ & 0.968 \\
\hline Tumor diameter, $\mathrm{cm}$ & $\leq 3$ vs. $>3$ & 1.293 & $0.735-2.274$ & 0.372 \\
\hline Helicobacter pylori infection & Negative vs. positive & 0.718 & $0.349-1.478$ & 0.368 \\
\hline Histological grade ${ }^{a}$ & 3 vs. 1 and 2 & 2.159 & $1.244-3.747$ & 0.006 \\
\hline Tumor stage & I vs. II & 1.794 & $0.973-3.308$ & 0.061 \\
\hline sPLA2-II expression & Low vs. high & 2.102 & $1.020-4.332$ & 0.044 \\
\hline
\end{tabular}

B, Multivariate analysis

\begin{tabular}{|c|c|c|c|c|}
\hline Parameter & Variable & Hazard ratio & $95 \%$ confidence interval & P-value \\
\hline Age, years & $\leq 65$ vs. $>65$ & 0.656 & $0.214-1.887$ & 0.415 \\
\hline Gender & Male vs. female & 1.126 & $0.440-3.140$ & 0.820 \\
\hline Tumor diameter, $\mathrm{cm}$ & $\leq 3$ vs. $>3$ & 1.243 & $0.609-2.537$ & 0.550 \\
\hline Helicobacter pylori infection & Negative vs. positive & 1.095 & $0.455-2.635$ & 0.840 \\
\hline Histological grade ${ }^{a}$ & 3 vs. 1 and 2 & 1.557 & $0.872-2.932$ & 0.170 \\
\hline Tumor stage & I vs. II & 3.165 & $1.413-7.089$ & 0.005 \\
\hline sPLA2-II expression & Low vs. high & 2.735 & $1.104-6.776$ & 0.030 \\
\hline
\end{tabular}

sPLA2-II, secretory phospholipase A-Group IIA. ${ }^{\mathrm{a}}$, well differentiated; 2 , moderately differentiated; 3 , poorly differentiated.

A

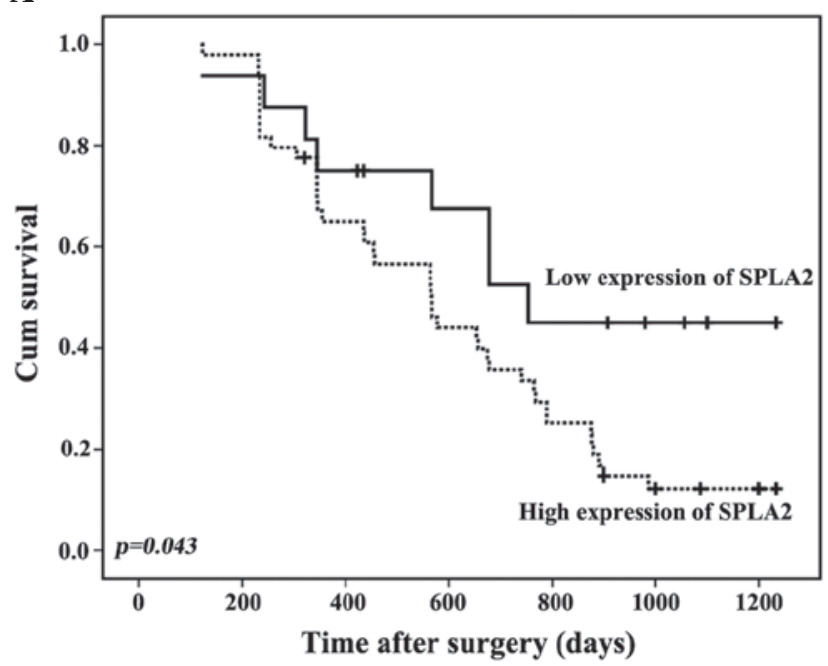

B

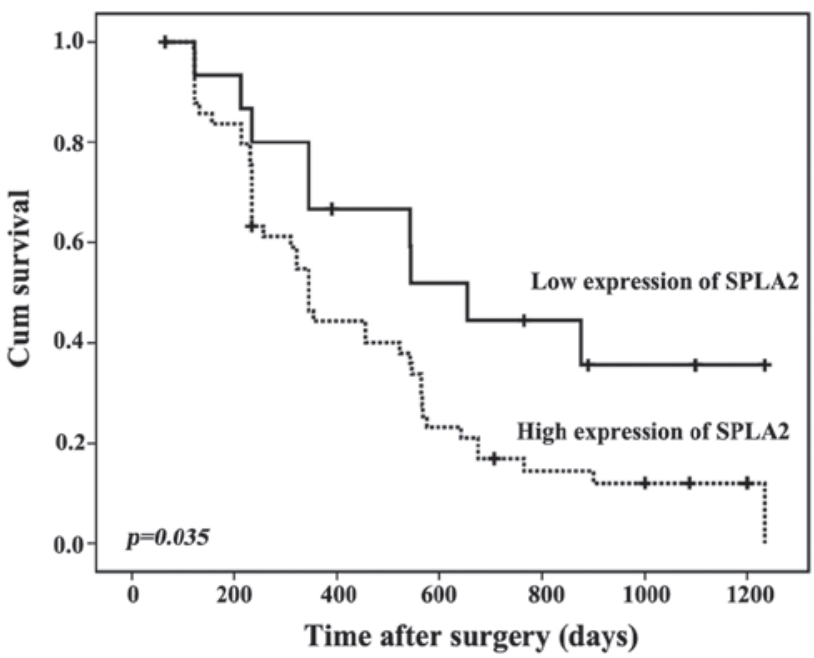

Figure 3. Kaplan-Meier survival curves indicate that patients exhibiting low sPLA2-II expression experience significantly improved OS and DFS (A) Kaplan-Meier curves for OS according to sPLA2-II expression. (B) Kaplan-Meier curves for DFS according to sPLA2-II expression. sPLA2-II, secretory phospholipases A-Group IIA; OS, overall survival; DFS, disease-free survival; cum, cumulative.

studies suggested that $H$. pylori infection may be associated with gastric cancer, and $H$. pylori has subsequently been identified as a World Health Organization class I carcinogen (20). $H$. pylori infection may damage the gastric mucosal barrier and stimulate epithelial cell proliferation and repair, which is associated with genetic mutations, abnormal gene expression, DNA damage and DNA methylation (21). The interaction between sPLA2-II expression and $H$. pylori remains to be elucidated, and further research may be required. It has been suggested that patients exhibiting gastric adenocarcinoma may benefit from anti-H. pylori therapy following surgery (22).

High sPLA2-II expression was observed not only in the present study, but also in previous studies. A previous study investigated the association between sPLA2-II expression and 
the characteristics of prostate cancer (23). Protein hybridization analysis was used to investigate sPLA2-II expression in benign prostate hyperplasia tissues. The results revealed that sPLA2-II expression was significantly increased in prostate cancer compared with benign prostatic hyperplasia. High sPLA2-II expression levels were also associated with malignancy (poorly differentiated primary prostate cancer tissues) (23). The results of this previous study were further confirmed by Jiang et al (24), who also observed that abnormally high sPLA2-II expression in prostate cancer was associated with malignancy. Another study evaluated sPLA2-II activity in 60 patients with hepatocellular carcinoma and demonstrated that sPLA2-II activity was increased in the carcinoma group compared with that of the control group (25). A study by Yamashita et al (26), which was based on preliminary test results in patients with various malignancies, revealed that increased plasma concentrations of sPLA2-II were associated with disease progression. In addition, following surgery, sPLA2-II levels returned to normal (26). Immunohistochemical studies of various types of cancer tissue, including pancreatic and breast cancer, have indicated positive expression of sPLA2-II $(27,28)$. Another study reported that serum sPLA2-II levels fell following tumor resection, suggesting that cancer cells may directly secrete sPLA2-II (29). This was supported by the results of an in vitro study (30). Thus, increased serum sPLA2-II may occur as a result of cancer cells inducing normal liver cells to produce sPLA2-II, or sPLA2-II may be directly produced by the cancer cells.

sPLA2-II may induce the development and progression of malignant tumors via production of arachidonic acid (23). Arachidonic acid levels are known to be increased in cancer tissues (31), and arachidonic acid is the raw material for the synthesis of inflammatory mediators, including prostaglandins and leukotrienes (32). These inflammatory mediators promote tumor formation, and sPLA2-II additionally stimulates the secretion of stromal cell growth factors and cytokines, which induce cell proliferation (33). It is possible that sPLA2-II produced by gastric cancer cells may also increase the arachidonic acid levels in tumor tissues, therefore enhancing the secretion of growth factors and cytokines, and thereby promoting cancer growth. This hypothesis is supported by the results of the present study, which indicated that poorly differentiated adenocarcinomas exhibit high sPLA2-II expression levels. The mechanisms underlying these phenomena remain to be elucidated.

In addition, the results of the present study revealed that high sPLA2-II expression was significantly correlated with reduced OS and DFS. According to the Cox proportional hazards regression multivariate analysis, high sPLA2-II was an independent risk factor of poor prognosis. However, previous studies have demonstrated differing results. sPLA2-II overexpression in human breast cancer is associated with disease severity, and the survival time of patients with high levels of sPLA2-II is reduced (34). In patients with prostate cancer, sPLA2-II expression is negatively correlated with 5-year survival rates (35). Studies of gastric adenocarcinoma have reached similar conclusions (36). Data from clinical studies suggests that sPLA2-II expression is a valuable prognostic indicator that may be applied to breast (37), esophageal (38), gastric (35), colon (39), liver (40) and pancreatic cancer (26). In clinical practice, high sPLA2-II levels may predict poor clinical outcomes; thus indicating the need for secondary screening of patients. The current study demonstrated that sPLA2-II may be a valuable indicator of gastric cancer development, progression and prognosis. Assays of serum sPLA2-II concentrations may aid in early diagnosis and the evaluation of treatment efficacy, and indicate prognosis of gastric cancer.

In addition, previous studies have demonstrated that sPLA2-II expression correlates with the invasiveness of cancer cells. sPLA2-II levels are increased in breast cancer tumors compared with those of benign breast tumors, and survival times are correspondingly reduced $(23,25,30,35)$. Immunohistochemical analysis revealed that sPLA2-II expression was increased in invasive pancreatic cancer and was associated with the presence of a larger number of cancer cells in the interstitial tissues $(16,35)$. sPLA2-II may act in combination with numerous oncogenes and tumor-suppressor gene mutations, which result in a variety of enzymatic changes. This may affect multi-gene, multi-factor and multi-step processes involved in oncogenesis, including genetic mutations, immunological and physiological biochemical changes, as well as hormonal abnormalities (29). sPLA2-II may possess a specific role in cancer development, expression and regulation, and an in-depth study of this role may reveal novel mechanisms underlying tumorigenesis.

Immunohistochemical methods have been utilized in order to elucidate the role of sPLA2-II in various types of cancer (29). However, an issue faced by researchers is the determination of the value of tumor immunohistochemical positivity for sPLA2-II that is clinically and biologically relevant. Previous studies have applied distinct scoring systems (29). It is difficult to identify a widely applicable methodology for evaluating sPLA2-II and its prognostic value in gastric adenocarcinoma. Therefore, the present study utilized a reproducible scoring method that considered staining percentage and intensity, and selected a cut-off score based on the ROC curve analysis method. Furthermore, sensitivity and specificity were high and the difference between them was small, leading to the greatest overall number of cases with the correct clinical diagnosis (41).

In conclusion, high sPLA2-II expression was observed in stage I and poorly differentiated cells, and these expression levels were significantly higher than those observed in stage II and poorly differentiated cells. sPLA2-II expression was not correlated with patient age, gender, tumor size or tumor location. sPLA2-II expression may be an adverse prognostic factor for gastric adenocarcinoma patients and there was an association between SPLA2-II overexpression and the histogenesis of gastric adenocarcinoma. The use of phospholipase inhibitors, including ulinastatin (42), or cyclooxygenase-2 inhibitors (43) in place of anti-H. pylori therapy following surgery, may reduce the risk of gastric cancer, by inhibiting tumor cell growth and promoting apoptosis of tumor cells, and therefore requires further study. However, further studies with increased cohorts of patients are required to verify this observation, and randomization and longer follow-up periods are required for the establishment of a safe and effective management plan based on this potentially significant molecular marker.

\section{Acknowledgements}

The present study was partially supported by Tianjin City High School Science \& Technology Fund Planning Project (grant 
no. 20110516), Tianjin Medical University Cancer Hospital Seed Fund Project (grant no. 0824), the National Natural Science Fund (grant no. 81101754), the National Science and Technology Support Project (grant no. 2012BAI15B06, Tianjin Science and Technology Support Plan Key Projects (grant no. 15ZCZDSY00890) and Tianjin Anti-Cancer Major Project (grant no. 12ZCDZSY20300).

\section{References}

1. Rasool S, Rasool V, Naqvi T, Ganai BA and Shah BA: Genetic unraveling of colorectal cancer. Tumour Biol 35: 5067-5082, 2014.

2. Arnold CN, Goel A, Blum HE and Boland CR: Molecular pathogenesis of colorectal cancer: Implications for molecular diagnosis. Cancer 104: 2035-2047, 2005.

3. Hayee B, Inoue H, Sato H, Santi EG, Yoshida A, Onimaru M, Ikeda $\mathrm{H}$ and Kudo SE: Magnification narrow-band imaging for the diagnosis of early gastric cancer: A review of the Japanese literature for the Western endoscopist. Gastrointest Endosc 78: 452-461, 2013

4. Clarke CJ, Forman S, Pritchett J, Ohanian V and Ohanian J: Phospholipase C-delta1 modulates sustained contraction of rat mesenteric small arteries in response to noradrenaline, but not endothelin-1. Am J Physiol Heart Circ Physiol 295: H826-H834, 2008.

5. Samuelsson B: Role of basic science in the development of new medicines: Examples from the eicosanoid field. J Biol Chem 287: 10070-10080, 2012.

6. D'Arrigo P and Servi S: Synthesis of lysophospholipids. Molecules 15: 1354-1377, 2010.

7. Liang NS and Fang ZW: Platelet phospholipase $\mathrm{A}_{2}$ and tumor. China Oncol 12: 555-558, 2002.

8. Hamilton JA: Stimulation of macrophage prostaglandin and neutral protease production by phorbol esters as a model for the induction of vascular changes associated with tumor promotion. Cancer Res 40: 2273-2280, 1980.

9. Wang M, Hao FY, Wang JG and Xiao W: Group IIa secretory phospholipase A2 (sPLA2IIa) and progression in patients with lung cancer. Eur Rev Med Pharmacol Sci 18: 2648-2654, 2014.

10. Dikken JL, van de Velde CJ, Gönen M, Verheij M, Brennan MF and Coit DG: The New American Joint Committee on Cancer/International Union Against Cancer staging system for adenocarcinoma of the stomach: Increased complexity without clear improvement in predictive accuracy. Ann Surg Oncol 19: 2443-2451, 2012.

11. Berlth F, Bollschweiler E, Drebber U, Hoelscher AH and Moenig S: Pathohistological classification systems in gastric cancer: Diagnostic relevance and prognostic value. World J Gastroenterol 20: 5679-5684, 2014.

12. Yousfi MM, el-Zimaity HM, Genta RM and Graham DY: Evaluation of a new reagent strip rapid urease test for detection of Helicobacter pylori infection. Gastrointest Endose 44: 519-522, 1996.

13. Zhu ZH, Sun BY, Ma Y, Shao JY, Long H, Zhang X, Fu JH, Zhang LJ, Su XD, Wu QL, et al: Three immunomarker support vector machines-based prognostic classifiers for stage IB non-small-cell lung cancer. J Clin Oncol 27: 1091-1099, 2009.

14. Constantine CE and Wreghitt TG: A rapid micro-agglutination technique for the detection of antibody to Legionella pneumophila serogroup 5. J Med Microbiol 34: 29-31, 1991.

15. Situ D, Wang J, Ma Y, Zhu Z, Hu Y, Long H and Rong T: Expression and prognostic relevance of MUC1 in stage IB non-small cell lung cancer. Med Oncol 28 (Suppl 1): S596-S604, 2011.

16. Zhao Jiuda, He Juxiang and Geng Paili: PLA2G II A gene in gastric cancer tissues and its significance. Shandong Medical Journal 46: 24-25, 2006.

17. Huhtinen HT, Grönroos JM, Uksila J, Grass DS, Nevalainen TJ and Laine VJ: Experimental Helicobacter felis infection in transgenic mice expressing human group IIA phospholipase A2. Helicobacter 9: 408-416, 2004.

18. Huhtinen HT, Grönroos JO, Grönroos JM, Uksila J, Gelb MH, Nevalainen TJ and Laine VJ: Antibacterial effects of human group IIA and group XIIA phospholipase A2 against Helicobacter pylori in vitro. APMIS 114: 127-130, 2006.
19. Menschikowski M, Rosner-Schiering A, Eckey R, Mueller E, Koch R and Jaross W: Expression of secretory group IIA phospholipase $\mathrm{A}(2)$ in relation to the presence of microbial agents, macrophage infiltrates, and transcripts of proinflammatory cytokines in human aortic tissues. Arterioscler Thromb Vasc Biol 20: 751-762, 2000.

20. Pandey R, Misra V, Misra SP, Dwivedi M, Kumar A and Tiwari BK: Helicobacter pylori and gastric cancer. Asian Pac J Cancer Prev 11: 583-588, 2010.

21. Hishida A, Matsuo K, Goto Y and Hamajima N: Genetic predisposition to Helicobacter pylori-induced gastric precancerous conditions. World J Gastrointest Oncol 15: 369-379, 2010.

22. Fock KM, Talley N, Moayyedi P, Hunt R, Azuma T, Sugano K, Xiao SD, Lam SK, Goh KL, Chiba T, et al; Asia-Pacific Gastric Cancer Consensus Conference: Asia-Pacific consensus guidelines on gastric cancer prevention. J Gastroenterol Hepatol 23: 351-65, 2008.

23. Ren P, Zhang JG, Xiu L and Yu ZT: Clinical significance of phospholipase A2 group IIA (PLA2G2A) expression in primary resected esophageal squamous cell carcinoma. Eur Rev Med Pharmacol Sci 17: 752-757, 2013.

24. Jiang J, Neubauer BL, Graff JR, Chedid M, Thomas JE, Roehm NW, Zhang S, Eckert GJ, Koch MO, Eble JN and Cheng L: Expression of group IIA secretory phospholipase A2 is elevated in prostatic intraepithelial neoplasia and adenocarcinoma. Am J Pathol 160: 667-671, 2002.

25. Liao Z, Fu X, Liao XS, Wang SQ and Kang GF: The change of serum phospholipase A2 activity and its clinical significance in patient with primary liver cancer. Chongqing Medical Journal 3: 203-204, 2000 (In Chinese).

26. Yamashita S, Ogawa M, Sakamoto K, Abe T, Arakawa H and Yamashita J: Elevation of serum group II phospholipase A2 levels in patients with advanced cancer. Clin Chim Acta 228: 91-99, 1994.

27. Cai H, Chiorean EG, Chiorean MV, Rex DK, Robb BW, Hahn NM, Liu Z, Loehrer PJ, Harrison ML and Xu Y: Elevated phospholipase A2 activities in plasma samples from multiple cancers. PLoS One 8: e57081, 2013.

28. Yamashita S, Yamashita J and Ogawa M: Overexpression of group II phospholipase A2 in human breast cancer tissues is closely associated with their malignant potency. Br J Cancer 69: 1166-1170, 1994.

29. Scott KF, Sajinovic M, Hein J, Nixdorf S, Galettis P, Liauw W, de Souza P, Dong Q, Graham GG and Russell PJ: Emerging roles for phospholipase A2 enzymes in cancer. Biochimie 92: 601-610, 2010.

30. Yamashita S, Yamashita J, Sakamoto K, Inada K, Nakashima Y, Murata K, Saishoji T, Nomura K and Ogawa M: Increased expression of membrane-associated phospholipase A2 shows malignant potential of human breast cancer cells. Cancer 71 : 3058-3064, 1993.

31. Kennedy BP, Soravia C, Moffat J, Xia L, Hiruki T, Collins S, Gallinger S and Bapat B: Overexpression of the nonpancreatic secretory group II PLA2 messenger RNA and protein in colorectal adenomas from familial adenomatous polyposis patients. Cancer Res 58: 500-503, 1998.

32. Kudo I and Murakami M: Phospholipase A2 enzymes. Prostaglandins Other Lipid Mediat 68-69: 3-58, 2002.

33. Fenard D, Lambeau G, Maurin T, Lefebvre JC and Doglio A: A peptide derived from bee venom-secreted phospholipase A2 inhibits replication of T-cell tropic HIV-1 strains via interaction with the CXCR4 chemokine receptor. Mol Pharmacol 60: 341-347, 2001.

34. Yamashita S, Yamashita J and Ogawa M: Overexpression of group II phospholipase A2 in human breast cancer tissues is closely associated with their malignant potency. Br J Cancer 69: 1166-1170, 1994.

35. Leung SY, Chen X, Chu KM, Yuen ST, Mathy J, Ji J, Chan AS, Li R, Law S, Troyanskaya OG, et al: Phospholipase A2 group IIA expression in gastric adenocarcinoma is associated with prolonged survival and less frequent metastasis. Proc Natl Acad Sci USA 99: 16203-16208, 2002.

36. Kiyohara H, Egami H, Kako H, Shibata Y, Murata K, Ohshima S, Sei K, Suko S, Kurano R and Ogawa M: Immunohistochemical localization of group II phospholipase A2 in human pancreatic carcinomas. Int J Pancreatol 13: 49-57, 1993.

37. Mannello F, Qin W, Zhu W, Fabbri L, Tonti GA and Sauter ER: Nipple aspirate fluids from women with breast cancer contain increased levels of group IIa secretory phospholipase A2. Breast Cancer Res Treat 111: 209-218, 2008 
38. Mauchley D, Meng X, Johnson T, Fullerton DA and Weyant MJ: Modulation of growth in human esophageal adenocarcinoma cells by group IIa secretory phospholipase A(2). J Thorac Cardiovasc Surg 139: 591-599, 2010.

39. Fijneman RJ and Cormier RT: The roles of sPLA2-IIA (Pla2g2a) in cancer of the small and large intestine. Front Biosci 13: 4144-4174, 2008.

40. Ikegami T, Matsuzaki Y, Fukushima S, Shoda J, Olivier JL, Bouscarel B and Tanaka N: Suppressive effect of ursodeoxycholic acid on type IIA phospholipase A2 expression in HepG2 cells Hepatology 41: 896-905, 2005.

41. Zlobec I, Steele R, Terracciano L, Jass JR and Lugli A: Selecting immunohistochemical cut-off scores for novel biomarkers of progression and survival in colorectal cancer. J Clin Pathol 60: 1112-1116, 2007.
42. Liu R, Qi H, Wang J, Wang Y, Cui L, Wen Y and Yin C: Ulinastatin activates the renin-angiotensin system to ameliorate the pathophysiology of severe acute pancreatitis. J Gastroenterol Hepatol 29: 1328-1337, 2014.

43. Hughes-Fulford M, Li CF, Boonyaratanakornkit J and Sayyah S: Arachidonic acid activates phosphatidylinositol 3-kinase signaling and induces gene expression in prostate cancer. Cancer Res 66: 1427-1433, 2006. 